\title{
ION DISTRIBUTION FUNCTIONS IN THE VICINITY OF COMET GIACOBINI-ZINNER
}

\section{T. E. Cravens}

Space Physics Research Laboratory, The University of Michigan, Ann Arbor, MI

Abstract. Photoionization of neutral molecules in the coma surrounding a comet produces heavy ions which contaminate and mass-load the solar wind. Cometary ion distribution functions in the vicinity of comet Giacobini-Zinner (G/Z) are calculated using a Monte Carlo method. The distribution function calculated behind the shock has both cold $(\approx 2 \mathrm{keV})$ and hot $(\approx 40 \mathrm{keV})$ components.

\section{Introduction}

The solar wind interacts very strongly with the very extensive atmospheres of comets (cf., Mendis et al., 1985; Galeev et al., 1985). The photoionization of cometary neutrals by solar radiation produces heavy ions (i.e., $\mathrm{H}_{2} \mathrm{O}^{+}$, $\mathrm{OH}^{+}, \mathrm{O}^{+}, \mathrm{CO}^{+}$, etc.) which are picked-up by the solar wind and mass-load it. The long plasma tails associated with the draping of magnetic field lines are one manifestation of this mass-loading (cf., Mendis et al., 1985) and the formation of a weak turbulent shock is another (cf., Galeev et al., 1985; Omidi et al., 1986; Sagdeev et al., 1986). The distribution function of the cometary ions picked up by the solar wind is, at least initially, highly anisotropic and non- Maxwellian (cf., Galeev et al., 1985; Sagdeev et al., 1986). A schematic of the solar wind interaction with comet $G / Z$ is shown in Figure 1 indicating the approximate locations of the shock and stagnation region.

The encounter of the ICE (previously ISEE-3) spacecraft with comet Giacobini-Zinner (G/Z) has provided the first in situ observations of the field and particle environment of a comet (see papers in the special Science issue, 1986). In particular, energetic ions (energies of the order of $100 \mathrm{keV}$ and greater) were detected more than $10^{6} \mathrm{~km}$ from the nucleus, and especially intense fluxes were seen in the "interaction region" near $10^{5} \mathrm{~km}$ (Hynds et al., 1986; Gloeckler et al., 1986). The Ion Composition Instrument (ICI) on ICE observed lower energy ions $(\approx 2 \mathrm{keV})$ at distances of about $1-4 \times 10^{4} \mathrm{~km}$ and actually identified several ion species including ions in the water "group" and $\mathrm{CO}^{+}$ (Oglivie et al., 1986).

Ideally, one would like to theoretically model the solar wind-cometary interaction by calculating the particle distributions and the fields (including wave fluctuations) selfconsistently and on a global scale. In practice, there are several methods: (1) self- consistent particle/field simulations but spatially local (e.g., Winske et al., 1985); quasi-linear methods are a variant of this (Sagdeev et al., 1986), (2) complete global MHD simulations but with no information concerning either non-Maxwellian particle distributions or field fluctuations (e.g., Fedder et al., 1986), (3) the semi-kinetic method which uses self-consistent particle distribution functions and fields, but which has been applied only along the stagnation line (sun-comet axis) (e.g., Wallis and Ong, 1975; Galeev et al., 1985), and (4) the Monte Carlo method in which large numbers of particle trajectories are calculated using an adopted large-scale model for the background fields. It is also possible to include field fluctuations in Monte Carlo calculations.

Copyright 1986 by the American Geophysical Union.

Paper number 6L6013.

0094-8276/86/006L-6013\$03.00
Some initial results of a Monte Carlo calculation will be presented in this paper. Valuable information can be derived on the details of the cometary ion distribution function using this method, although these distributions are not necessarily consistent with the background field model assumed. This particular paper employs a relatively simple background model taken from Galeev et al. (1985); however, more realistic and complicated models, including field fluctuations, will be used in future calculations.

\section{Model Description}

The flow velocity vector, $\mathbf{u}(\mathbf{x})$, in the supersonic solar wind upstream of the shock can be described as a function of the distance, $x$, from the nucleus on the sun-comet axis with the following expression (Biermann et al., 1967; Galeev et al., 1985):

$$
\text { with } \quad \rho \hat{u}=1+\frac{Q M}{4 \pi v_{g} \tau \rho_{\infty} u_{\infty}} \frac{1}{r}
$$$$
\hat{\mathrm{u}}=\frac{2}{3 \rho \hat{\mathrm{u}}}\left[1+(1-3 \rho \hat{\mathrm{u}} / 4)^{1 / 2}\right]
$$

pû is the mass flux normalized to that in the unperturbed solar wind far upstream, $p_{\infty} u_{\infty}$, and $\hat{\mathrm{u}}=\mathrm{u} / \mathrm{u}_{\infty}$ is a similarly normalized flow speed. $Q$ is the gas production rate of the comet (a value of $2.3 \times 10^{28} \mathrm{~s}^{-1}$ is used here for $G / Z$ at a

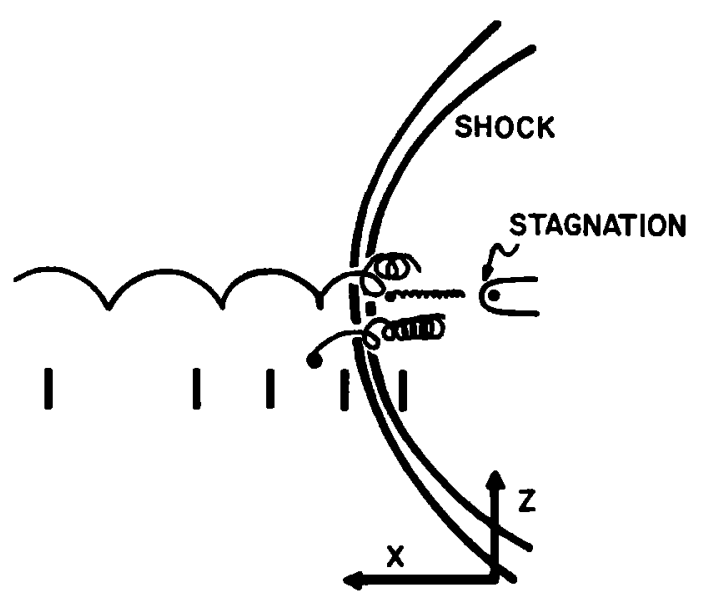

Fig. 1. Schematic of solar wind interaction with comet Giacobini-Zinner showing the approximate location of shock and stagnation region and a reasonable thickness for the shock (or interaction region). The actual shock position at the time of ICE encounter was somewhat closer to the nucleus (Fuselier et al., 1986). Three calculated trajectories are shown for which the flow field and shock position were assumed to be planar. The leftmost trajectory started $3 \times 10^{5} \mathrm{~km}$ upstream of the edge of the figure. The shock structure shown here is schematic. Locations of 5 "bins" along the $\mathrm{x}$-axis are indicated by vertical straight lines 
a.)

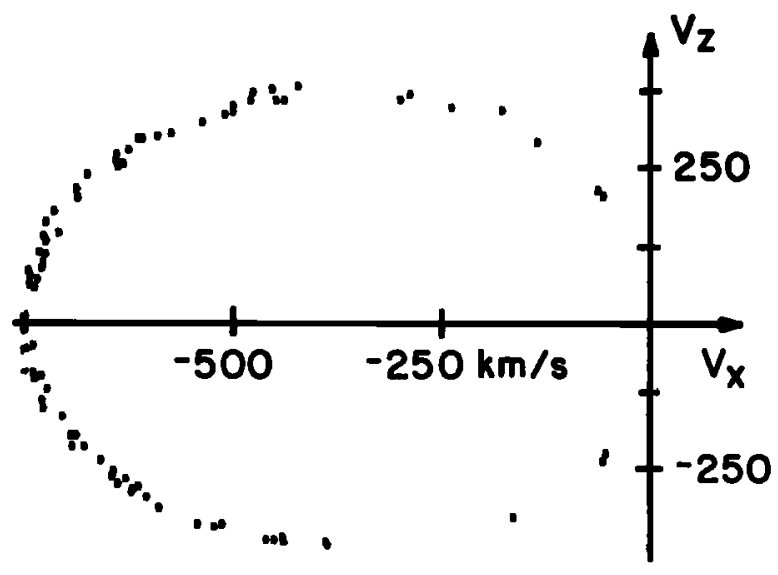

c.)

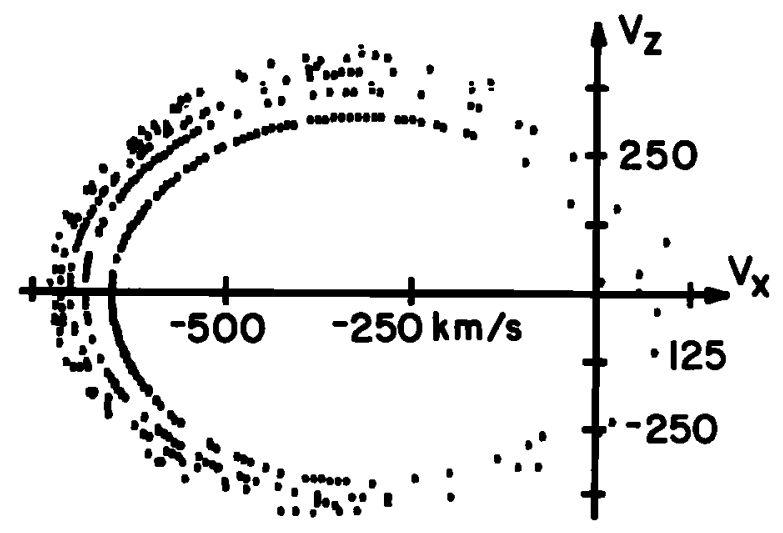

b.)
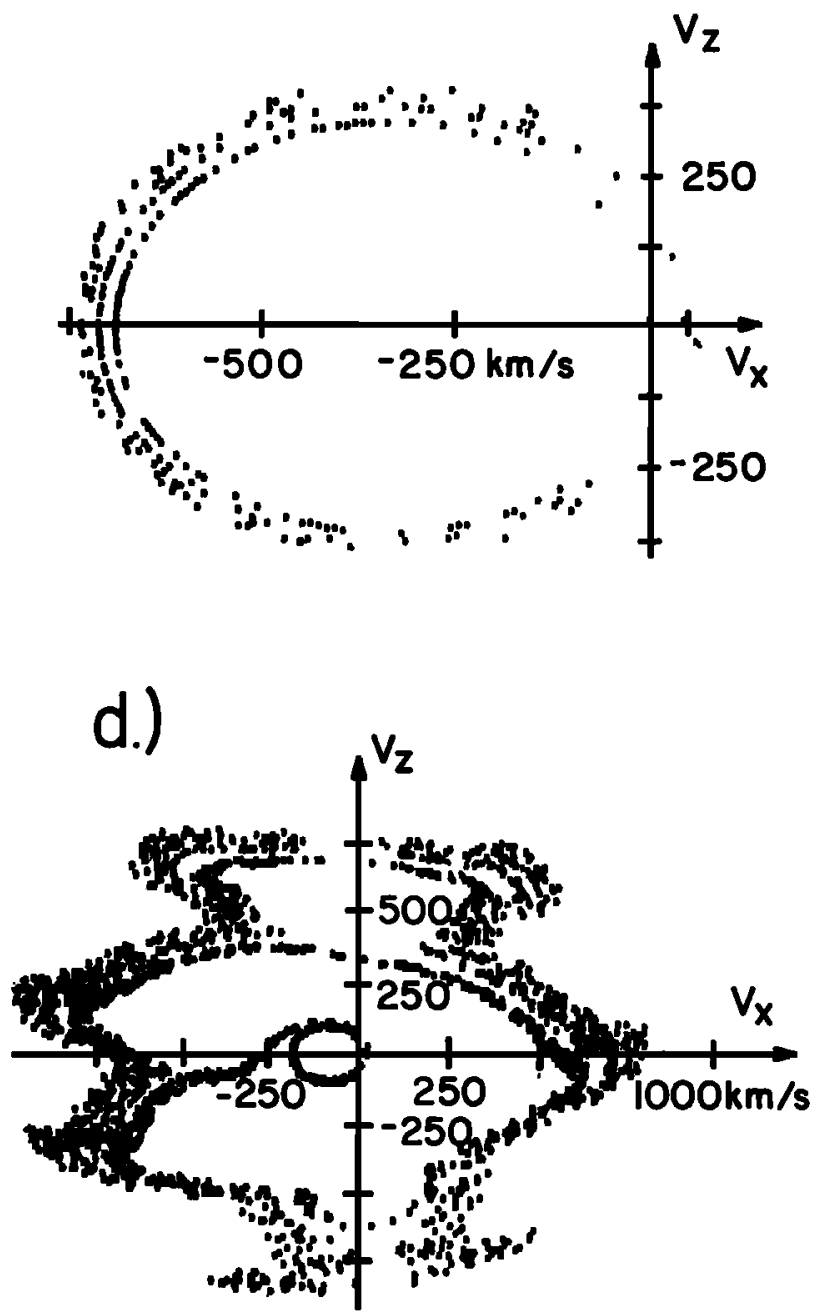

Fig. 2. Velocity space diagram in $v_{x}$ and $v_{\text {a }}$ for ions crossing plane surfaces at the following distances from the nucleus along the $x$-axis: (a) $3 \times 10^{3} \mathrm{~km}$, (b) $1.5 \times 10^{3} \mathrm{~km}$, (c) $1.0 \times 10^{5} \mathrm{~km}$, and (d) $0.6 \times 10^{5} \mathrm{~km}$. The last bin is downstream of the shock (in this model)

heliocentric distance of $1 \mathrm{AU}$ ), $M$ is the ion mass (18 amu is used here although far from the comet $M=16$ or 17 , that is, $\mathrm{O}^{+}$or $\mathrm{OH}^{+}$, would be more appropriate $), \mathrm{vg}(\approx 1 \mathrm{~km} / \mathrm{s})$ is the neutral outflow speed, and $\tau\left(\approx 10^{6} \mathrm{~s}\right)$ is the ionization lifetime. An unperturbed solar wind density of $n_{\infty}=5 \mathrm{~cm}^{-3}$ and speed of $u_{\infty}=400 \mathrm{~km} / \mathrm{s}$ are assumed. These solar wind parameters are similar but not the same as those measured by ICE at the time of the encounter. The effects of the attenuation of the neutral density due to photoionization is neglected in equation (1). Values of $u$ from (1) and from the MHD model of Fedder et al. (1986) are in close agreement along the sun-comet axis. The magnetic field in the unperturbed solar wind is taken to be in the $y$ direction with a magnitude of $B_{\infty}=5 \mathrm{nT}$. The field closer to the nucleus is found from $\mathrm{uB}=$ $u_{\infty} B_{\infty}$. The solar wind electric field is just $\mathbf{E}=-\mathbf{u} x \mathbf{B}$, or $E$ $=\mathrm{u}(\mathbf{x}) \mathbf{B}(\mathbf{x})$.

The shock parameters were adopted from Galeev et al. (1985). A Mach number 2 shock of finite width is placed between 0.8 and $0.9 \times 10^{5} \mathrm{~km}$. The flow speeds just ahead and just behind the shock are $300 \mathrm{~km} / \mathrm{s}$ and $107 \mathrm{~km} / \mathrm{s}$, respectively. B within the shock is assumed to vary linearly from its preshock to its postshock value. $u$ in the shocked solar wind is given by the gasdynamic expressions in Galeev et al.(1985).

1000 ions of mass $M$ are created randomly with zero initial velocity and with a probability proportional to $1 / r^{2}$ where $r$ is the radial distance from the comet. Only ions created within the rather narrow yolume $\Delta y=\Delta z= \pm 0.3 \times 10^{5} \mathrm{~km}$ and $6 \times 10^{5}$ $\mathrm{km} \leq \mathrm{x} \leq 0.2 \times 10^{5} \mathrm{~km}$ are considered because of the one dimensional dependence of the $E$ and $B$ fields on $x$ and because $u$ and $B$ are orthogonal. The trajectory for each ion is determined by numerically solving the equation of motion:

$$
\mathrm{dx} / \mathrm{dt}=\mathbf{v} \quad \mathbf{M d v} / \mathrm{dt}=\mathrm{q}(\mathbf{E}+\mathbf{v} \times \mathbf{B})
$$

where $q$ is the charge, $x=(x, y, z)$ is the position vector, and $v$ is ion velocity vector.

\section{Results}

Three sample trajectories are shown in Figure 1. The ion born far upstream of the shock (off the figure to the left) executes cycloidal motion as expected-- drifting with the solar wind velocity $u$ and also gyrating with perpendicular velocity 


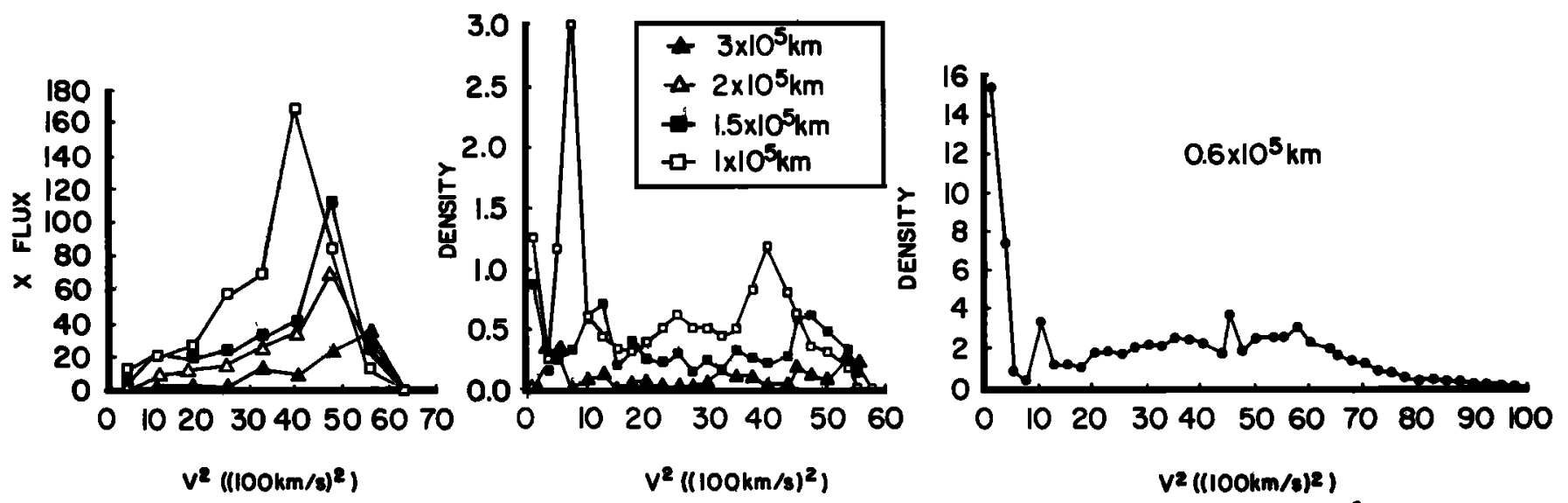

Fig. 3. (left panel) Omni-directional flux in $X$-direction (in arbitrary units) versus velocity squared. $V^{2}$ is proportioned to the total energy. $V^{2}=50 \times 10^{4}(\mathrm{~km} / \mathrm{s})^{2}$ corresponds to $43 \mathrm{keV}$ for water ions. (Middle and right panels): Density distributions versus $\mathrm{V}^{2}$. The same arbitrary units are used in both of these panels, hence the total integrated density for the subsonic bin (right) is much greater than for the bins located upstream of the shock.

$v_{\perp}=u$. The Larmor radius, $r_{L}$, for this heavy water ion (or an oxygen ion) is extremely large - approximately $2 \times 10^{4} \mathrm{~km}$. $\mathrm{CO}^{+}$ions have even larger Larmor radii. As the ion drifts towards the comet, the solar wind is gradually slowing down and the ion motion is no longer exactly cycloidal. The magnetic moment, $\mu=\mathrm{M} \mathrm{v}_{\perp}{ }^{2} / 2 \mathrm{~B}$, is conserved except near the shock. Field fluctuations are not included in this preliminary model so that the parallel velocity along $B$ always remains zero -- that is, there is no isotropization.

The maximum velocity occurs at the top of the arc of the cycloid and the maximum total energy is $T_{\max }=4 T_{\perp} \sin ^{2} \alpha=$ $2 \mathrm{Mu}^{2} \sin ^{2} \alpha$, where $\mathrm{T}_{1}$ is the perpendicular energy and $\alpha$ is the angle between the solar wind and magnetic field direction (Hynds et al., 1986). $\alpha$ is $90^{\circ}$ for this calculation. $T_{\text {max }}$ for the $\mathrm{H}_{2} \mathrm{O}^{+}$trajectory shown in Figure 1 is $60 \mathrm{keV}$; it would be $93.2 \mathrm{keV}$ for a $\mathrm{CO}^{+}$ion with $\mathrm{u}=400 \mathrm{~km} / \mathrm{s}$.

The ion trajectory undergoes a sudden drift in the $+z$ direction at the shock (Figure 1) due to the sharp gradient in the magnetic field strength. The ion gains energy, $\Delta T=q E \Delta z$ $\approx \mathrm{q} \operatorname{luB}_{\mathrm{r}} \approx \mathrm{Mu}^{2}$, due to its drift along the direction of $\mathbf{E}$. In general, the magnitude of $\Delta \mathrm{T}$ depends on the phase at which the ion first encounters the shock. The post-shock ion continues to drift towards the comet with $\mathrm{u} \approx 100 \mathrm{~km} / \mathrm{s}$ and $\mathrm{v}_{\perp} \approx 800 \mathrm{~km} / \mathrm{s}$.

Another ion (Figure 1) is born just ahead of the shock. It too experiences some acceleration at the shock but is not quite as energetic as the first ion. A third sample trajectory starts in the shocked solar wind and initially executes cycloidal motion with $v_{1} \approx u=100 \mathrm{~km} / \mathrm{s}$; this ion is much less energetic than the other two ions and belongs to the ion population that the ICI instrument on ICE (Ogilvie et al.,1986) is capable of observing.

Particle distribution functions were calculated at 5 planar surfaces (i.e., $x=0.6,1.0,1.5,2.0$, and $3.0 \times 10^{5} \mathrm{~km}$ ). Velocity space diagrams $\left(v_{x}, v_{z}\right)$ are shown for four of these "bins" in Figure 2. In the relatively unperturbed solar wind $\left(x=3 \times 10^{5} \mathrm{~km}\right.$, Figure $\left.2 \mathrm{a}\right)$ the points are distributed in a circular (it appears elliptical in the figure) ring with radius $v_{\perp}=$ $\mathrm{u}=380 \mathrm{~km} / \mathrm{s}$ and with a displacement along the $\mathrm{v}_{\mathrm{x}}$ axis of $-380 \mathrm{~km} / \mathrm{s}$. There are fewer points near $v_{x}=0$ since the probability of a particle being at a given location on this diagram is proportional to the flux in the $x$-direction, $F_{x}$, which goes to zero at $v_{x}=0$. The distributions closer to the comet (Figures $2 \mathrm{~b}, \mathrm{c}$ ) are somewhat more complicated. For instance, at $x=1.0 \times 10^{5} \mathrm{~km}$, there are several rings, all displaced along the $v_{x}$ axis by $u=-330 \mathrm{~km} / \mathrm{s}$. The innermost ring is due to ions born not far upstream of this bin and has radius $v_{\perp}=u=330 \mathrm{~km} / \mathrm{s}$. The outer ring is due to ions born much further upstream and has $v_{\perp}=418 \mathrm{~km} / \mathrm{s}$, which is larger than even $u_{\infty}$ because of the conservation of the adiabatic invariant $\mu$.

The quantization of the rings is real and not just a numerical artifact, although this is rather academic since magnetic fluctuations will tend to smooth them out. Actually, the loci of points is one spiral which starts at the origin (ions botn exactly at the bin in question) and goes in a counterclockwise direction, representing ions born further and further upstream. For example, for the phase angle corresponding to maximum $\left|v_{x}\right|$, the successive rings represent ions born at certain discrete times upstream of any given bin: $\Delta t \cong-(n / 2)\left(2 \pi / \Omega_{\mathrm{Hi}}\right)$ with $n=1,3,5, .$. and where $\Omega_{\mathrm{Hi}}$ is the gyrofrequency. Ions with larger $\Delta t$ are born further upstream where $u$ is larger and hence have larger values of $v_{1}$. The rings of the spiral tend to merge together for large vatues of $n$, since far upstream $u$ becomes the unperturbed solar wind speed.

The velocity diagram for the shocked ions $\left(x=0.6 \times 10^{5} \mathrm{~km}\right.$, Figure 2d) is rather complicated but there are clearly two major populations (see Galeev et al.,1985): (1) a low energy population due to ions born downstream of the shock for which both $v_{\perp}$ and $x$-drift are equal to $u \approx 100 \mathrm{~km} / \mathrm{s}$, and (2) a high energy population due to ions born in the unshocked solar wind and carried through the shock. These "hot" ions also have drift velocity u but have $v_{1}=700-900 \mathrm{~km} / \mathrm{s}$. Many of these hot ions have been accelerated at the shock as described earlier. In addition, there is an intermediate population due to ions born within the shock. The distribution of the"old", and energetic, ions is quite complicated and its details should not be taken too seriously since the real distribution will depend on the exact structure of the shock as well as on the degree of isotropization taking place due to scattering by magnetic fluctuations (Sagdeev et al.,1986). However, whatever the detailed nature of the shock both hot and cold cometary ion populations certainly exist in the magnetosheath as has been confirmed by ICE observations of at least portions of these two populations by Hynds et al. (1986) and Oglivie et al. (1986), respectively.

The information collected at each bin was also used to calculate a variety of distribution functions, some of which are shown here. The distribution of both the density, $\mathrm{dn} / \mathrm{dV}^{2}$, and the ion flux in the $x$-direction, $F_{x}$ are shown as functions of the total velocity squared (i.e., energy) in Figure 3 . The distribution functions for the supersonic solar wind can be 
understood in terms of the convolution of ring distributions taking into account the appropriate Jacobean. As expected, both $\mathrm{dn} / \mathrm{dV}^{2}$ and $F$ increase with decreasing $x$. These calculated distributions are all highly anisotropic since pitch-angle scattering was neglected. Far from the nucleus this is in agreement with observations, but in the vicinity of the interaction region the measured distributions appear to be isotropized (Hynds et al., 1986; Gloeckler et al., 1986). Furthermore, the calculated distributions in the solar wind frame of reference (not shown in Figure 3) have both low and high energy cutoffs, whereas the Gloeckler et al. (1986) measurements suggest that if there is a low energy cutoff it is considerably lower than what the calculations here would predict.

The density distribution in the shocked solar wind has the hot and cold components discussed earlier (Figure 3c). The hot component (with ion energies about $40 \mathrm{keV}$ ) has a high energy tail associated with shock acceleration. As Galeev et al. (1985) pointed out, the cold component (ion energies less than $2 \mathrm{keV}$ ) continues to grow as $\mathrm{x}$ decreases and it contains most of the mass, although the hot component accounts for most of the pressure. The hot component is expected to decay near the stagnation region because the hot ions will escape from this region along the field lines and also because these ions will charge exchange with neutrals. These decay processes were not included in the current version of the Monte Carlo Model.

\section{Summary}

Cometary ion distributions were determined at several locations in the vicinity of comet $G / Z$ using a Monte Carlo method in which 1000 trajectories were calculated. The background electric and magnetic fields were taken from a relatively simple model appropriate for the stagnation line. The next step in the development of this method is to employ a more realistic model for the average fields, such as the Fedder et al. (1986) MHD model. Magnetic field fluctuations should also be included to allow for pitch angle scattering and isotropization of the picked-up ions in the vicinity of the shock (or interaction region), as suggested by ICE measurements of ion distribution functions (Gloeckler et al., 1986). Finally, the results of the Monte Carlo model should be carefully compared with measurements made by instruments on the spacecraft flown to comets $G / Z$ and Halley (e.g., Ogilvie et al., 1986).

Acknowledgments. This work was supported by NASA grants NAGW-15 and NAG-5-565 (ICE guest investigator) and NSF grant ATM 8417884 . The author thanks C. Ford, R. Nightingale, and C. Laidlaw for their computational assistance.

\section{References}

Biermann, L, B. Brosowski, and H.V. Schmidt, The interaction of the solar wind with a comet, Solar Phys, 1 , 254-283, 1967.

Fedder, J.A., J.G. Lyon, and J.L. Giuliani, Jr., Numerical simulations of comets: Predictions for comet Giacobini-Zinner, Trans. Am. Geophys Union, 67, 17, 1986.

Fuselier, S.A., W.C. Feldman, S.J. Bame, E.J. Smith, and F.L. Scarf, Heat flux observations and the location of the transition region of Giacobini-Zinner, Geophys. Res. Lett., This issue, 1986.

Galeev, A.A., T.E. Cravens, and T.I. Gombosi, Solar wind stagnation near comets, Astrophys. I.,289, 807-819, 1985.

Gloeckler, G., D. Hovestadt, F.M. Ipavich, M. Scholer, B. Klecker, and A.B. Galvin, Cometary pick-up ions observed near Giacobini-Zinner, Geophys. Res. Lett., This issue, 1986.

Hynds, R.J., S.W.H. Cowley, T.R. Sanderson, J.J. VanRuoijen, and V.-P. Wenzel, Observations of energetic ions from comet Giacobini-Zinner, in press, Science, 1986.

Mendis, D.A., H.L.F. Houpis, and M.L. Marconi, The physics of comets, Eundamentals of Cosmic Physics, 10 , $1,1985$.

Ogilvie, K.W., M.A. Coplan, P. Bochler, and J. Geiss, Results from the ion composition instrument during the encounter of ICE with Giacobini-Zinnet, Science, 1986.

Omidi, N., D. Winske, and C.S. Wu, The effect of heavy ions on the formation and structure of cometary bow shocks, in press, Icarus, 1986.

Sagdeev, R.Z., V.D. Shapiro, V.I. Shevchetko, and K. Szego, MHD turbulence in the solar wind--Comet interaction region, in press, Geophys. Res. Lett., 1986.

Wallis, M.K. and R.S. B. Ong, Strongly cooled ionizing plasma flows with application to beams, Planet Space Sci., 23, 713-720, 1975.

Winske, D., C.S. Wu, Y.Y. Li, Z.Z. Mou, and S.Y. Guo, Coupling of newborn ions to the solar wind by electromagnetic instabilities and their interaction with the bow shock, L. Geophys. Res, 90, 2713-2727, 1985.

T.E. Cravens, Space Physics Research Laboratory, The University of Michigan, Ann Arbor, MI 48109.

(Received January 16, 1986;

Accepted January 28, 1986.) 